Archives de sciences sociales des religions

114 | avril-juin 2001

Varia

\title{
Rapports entre le religieux et le politique en Amérique latine
}

\section{Michael Löwy}

\section{CpenEdition}

Journals

Édition électronique

URL : http://journals.openedition.org/assr/20728

DOI : $10.4000 /$ assr.20728

ISSN : $1777-5825$

Éditeur

Éditions de l'EHESS

Édition imprimée

Date de publication : 1 juin 2001

Pagination : 61-66

ISBN : 2-222-96704-X

ISSN : 0335-5985

Référence électronique

Michael Löwy, "Rapports entre le religieux et le politique en Amérique latine », Archives de sciences sociales des religions [En ligne], 114 | avril-juin 2001, mis en ligne le 19 août 2009, consulté le 10 décembre 2020. URL : http://journals.openedition.org/assr/20728; DOI : https://doi.org/10.4000/ assr.20728

Ce document a été généré automatiquement le 10 décembre 2020.

(C) Archives de sciences sociales des religions 


\title{
Rapports entre le religieux et le politique en Amérique latine
}

\author{
Michael Löwy
}

\section{RÉFÉRENCE}

DROGUS (Carol Ann), Women, Religion and Social Change in Brazil's Popular Church, Notre Dame, University of Notre Dame Press, 1997, 226 p.

KLAIBER (Jeffrey S.J.), The Church, Dictatorships and Democracy in Latin America, Maryknoll, Orbis Books, 1998, $326 \mathrm{p}$.

SMITH (Brian H.), Religious Politics in Latin America. Pentecostal vs. Catholic, Notre Dame, University of Notre Dame Press, 1998, 126 p.

SMITH (Christian), PROKOPY (Joshua), eds., Latin American Religion in Motion, New York, Routledge, 1999, $303 \mathrm{p}$.

CENTRE TRICONTINENTAL DE LOUVAIN-LA-NEUVE, Théologies de la libération, Alternatives Sud. Paris, L'Harmattan, 2000, $312 \mathrm{p}$.

1 Après avoir été pendant des siècles le gardien le plus fidèle des principes d'Autorité, d'Ordre et de Hiérarchie, l'Église catholique latino-américaine - ou mieux, une partie de celle-ci - est devenue, presque sans transition, une force sociale critique, un pôle d'opposition aux régimes autoritaires, un pouvoir contestataire de l'ordre établi. Cela a pris, au cours des années soixante-dix et quatre-vingt la forme d'une spectaculaire confrontation entre l'Église comme institution et un certain nombre d'États gouvernés par des dictatures militaires - une confrontation qui a joué un rôle déterminant dans la crise de légitimité de ces régimes. Ce n'est pas étonnant que ce phénomène sans précédent ait attiré l'attention de scientifiques sociaux: sociologues et politologues se sont penchés sur cette forme nouvelle de rapport entre le religieux et le politique, en essayant de la comprendre et de l'analyser. Aujourd'hui, ce sont les historiens qui prennent la relève et qui proposent un bilan de ce chapitre inédit de l'histoire de l'Église. 
2 C'est le cas, par exemple, du volume récemment paru aux États-Unis, rédigé par un jésuite américain, J. Klaiber, qui dirige depuis de longues années le département de sciences humaines de l'Université Catholique de Lima. L'auteur examine, pays par pays - Brésil, Chili, Argentine, Paraguay, Uruguay, Bolivie, Pérou, El Salvador, Nicaragua, Guatemala et Mexique - le rôle des Églises, ou de secteurs des Églises, dans l'opposition aux dictatures militaires et dans la lutte pour la redémocratisation. C'est une synthèse bien utile, solidement documentée et qui permet effectivement une vue d'ensemble de la question. Le point de vue de l'auteur est modéré, et il met plus volontiers en avant le rôle médiateur et pacificateur de l'Église que celui, plus « subversif », des religieux ou des laïcs engagés dans les luttes sociales; mais il n'occulte pas les divergences au sein de l'institution entre traditionalistes et progressistes, ainsi que le rôle foncièrement conservateur du Vatican sous Jean Paul II.

On est frappé, en lisant cet ouvrage, par le fait que dans chaque pays, malgré les énormes différences sociales, politiques, culturelles et religieuses, il a existé, au sein de l'Église, un courant qui s'est engagé de façon conséquente pour la démocratie, pour les droits de l'homme, pour la cause des classes subalternes - quitte à souffrir de la répression, de l'emprisonnement ou de la mort. Mais il est non moins évident que l'Église, en tant qu'institution, c'est-à-dire les confédérations d'évêques de chaque pays, est loin d'avoir réagi de la même façon : tandis qu'au Brésil, après 1970, l'Église catholique est devenue, pendant une quinzaine d'années, la principale force d'opposition au régime militaire, en Argentine la majorité des évêques a apporté un soutien sans faille à la dictature et à sa « guerre sale » contre la "subversion ». Les Églises des autres pays se situent entre ces deux pôles. L'auteur prend note de ces différences, mais les explications qu'il propose ne sont pas toujours convaincantes. Ainsi, il tente de rendre compte du rôle de l'Église brésilienne par l'absence d'un grand parti démocrate chrétien, dont la place aurait été occupée par les mouvements catholiques laïcs. Le cas argentin serait à expliquer lui aussi par... l'absence d'un grand parti démocrate chrétien, qui aurait incité l'Église à s'adresser directement aux militaires. Il est évident que le même facteur ne peut pas rendre compte des radicales différences de comportement entre les deux Églises. En fait, ce qui manque dans le livre en question c'est une réelle analyse comparative, qui puisse expliquer la posture des différentes Églises face à l'État et aux régimes d'exception. Quelques hypothèses sont esquissées dans l'introduction: au Mexique, en Argentine et en Colombie, le conflit avec des gouvernements libéraux a renforcé la tendance intégriste dans l'Église, tandis qu'à Cuba, en Haïti et en Uruguay, l'Église était trop faible pour jouer un rôle politique important. Le cas brésilien n'entre dans aucune de ces typologies. L'auteur examine aussi les influences extérieures, notamment celle de l'Église nordaméricaine sur celles des pays d'Amérique centrale - plutôt favorable au courant progressiste modéré. Mais il ne mentionne nulle part l'influence importante du catholicisme français en Amérique latine.

4 Cet ouvrage, qui a le grand mérite d'être la première vue d'ensemble de l'histoire des conflits entre les Églises et les dictatures en Amérique latine, souffre aussi d'une excessive confiance dans l'utilité du paradigme dualiste "tradition vs. modernité ». Ainsi, il explique la faiblesse de la démocratie en Amérique latine par des "survivances coloniales » et définit le conflit au sein des Églises comme opposant traditionalistes et modernistes. Il me semble que la réalité est plus complexe, dans la mesure où les dictatures militaires avaient un programme de modernisation économique autoritaire et que les chrétiens libérationistes ne cachaient pas leurs critiques à la modernité 
capitaliste/industrielle. Cela permet de mieux comprendre la conclusion de l'ouvrage, où l'auteur se réfère à la déception des chrétiens progressistes avec l'orientation néolibérale qui a prédominé en Amérique latine après la restauration de la démocratie.

5 Cette question est importante : l'opposition au néolibéralisme correspond à une tradition ancienne de l'Église, qui continue à nourrir la contestation des logiques économiques dominantes en Amérique latine par de larges secteurs de l'Église au cours des années quatre-vingt-dix. Curieusement, c'est une problématique pratiquement absente de la littérature scientifique récente, notamment d'origine nord-américaine, sur les Églises latino-américaines. C'est le cas, par exemple, du recueil organisé par Christian Smith et Joshua Prokopy sur les tendances religieuses actuelles du continent. Aucune des nombreuses contributions de cet ouvrage collectif ne traite de cette question: le thème du néolibéralisme semble absent - sauf dans un des articles, celui de Sarah Brooks sur les nouvelles stratégies catholiques à l'époque du néolibéralisme, qui se limite cependant à constater que les réformes néolibérales sont la condition d'une économie efficace et que les militants catholiques doivent s'adapter à ce nouveau environnement, par exemple en s'associant à des entreprises multinationales pour mener des campagnes sociales.

Le thème dominant de ce recueil est celui du pluralisme religieux en Amérique latine : les deux organisateurs constatent, avec raison, que cette diversité de l'offre religieuse n'a pas provoqué, comme le suggéraient certaines des théories de la sécularisation, un déclin de la foi, bien au contraire. Malheureusement, ils essayent - ainsi que certains autres auteurs du recueil - d'analyser ce pluralisme à travers la grille de lecture fournie par la théorie des "économies religieuses" de Roger Finke et Rodney Stark : le champ religieux est assimilé, de la façon la plus plate possible, à un marché de biens de consommation, analysé avec les concepts typiques des doctrines économiques libérales. Le conflit entre dénominations est ainsi réduit à un affrontement entre les partisans (catholiques) du monopole étatique de la production de biens spirituels et leurs rivaux (pentecostaux) favorables à la déréglementation du marché et à la saine concurrence entre les entreprises. Les difficultés qu'affronte l'Église catholique dans ce combat pour la clientèle résulteraient du fait que les monopoles nourrissent un clergé paresseux...

7 On retrouve la même méthode dans la contribution d'Anthony Gill sur les réponses catholiques à la croissance du protestantisme en Amérique latine. L'Église catholique doit, selon cet auteur, être assimilée à une entreprise dont l'intérêt principal est la maximisation des parts de marché (plutôt que le profit financier), et dont la stratégie est celle des industries de style ancien qui cherchent à s'imposer grâce à des tarifs protectionnistes et des subsides publics. Certes, reconnaît l'auteur, les biens religieux par exemple le salut éternel - ne sont pas tout à fait du même type que les autres : ce sont des «biens de croyance », des marchandises dont le client ne peut pas définir la qualité ni avant ni après l'achat, et dont le prix est difficile à déterminer. L'avenir appartient, bien entendu, à ceux qui préconisent un marché ouvert et compétitif...

8 Heureusement, tous les papiers de ce volume ne sont pas de cette eau là, et nous offrent une série d'analyses intéressantes sur les rapports actuels entre religion et politique. Liesl Haas examine l'évolution de l'Église chilienne, qui, après avoir combattu le régime militaire du général Pinochet et défendu les droits de l'homme, s'est vouée, au cours des dernières années, à la cause de la lutte contre le divorce et contre l'éducation sexuelle dans les écoles - en alliance avec les anciens partisans du même général. Analysant les pratiques politiques des Églises pentecôtistes chiliennes, Frans Kamsteeg montre que celles-ci se sont divisées en deux blocs nettement distincts : le Conseil Pastoral, fondé en 
1975, qui a soutenu sans états d'âme le régime militaire qui a « sauvé le Chili de la force satanique du Marxisme » et la Confraternité Chrétienne des Églises, fondée en 1985, qui a pris position contre le régime d'exception et pour les droits de l'homme.

9 L'influence du pentecôtisme s'est exercé aussi au sein de l'Église catholique, par l'essor des charismatiques. Selon Marjo de Theije, contrairement à ce que l'on prétend souvent, les CEBs et les groupes charismatiques au Brésil sont loin de s'opposer: les fidèles participent souvent aux deux et leurs activités sur le terrain local se recoupent partiellement. Le même vaut, mutatis mutandis, pour les initiatives de l'Église progressiste et les pratiques religieuses populaires, étudiés par Kris tin Norget à partir d'une recherche sur le terrain à Oaxaca, Mexique. Selon ce papier, sans doute un des plus intéressants du recueil, la religion populaire - considérée avec une certaine méfiance par le clergé progressiste - est un ensemble de pratiques inspirées par un code éthique populaire qui valorise la coopération, la solidarité, l'échange, la réciprocité. Loin d'être une simple survivance du passé, il s'agit d'une relation sociale active, la voix d'une identité populaire qui s'enracine dans des traditions locales anciennes (idéalisées), et qui s'oppose au discours dominant de la modernité et du progrès.

Pendant longtemps, les travaux sur religion et politique en Amérique latine se sont penchés presque exclusivement sur l'Église catholique; depuis le milieu des années quatre-vingt, ce sont plutôt les Églises pentecôtistes qui ont monopolisé l'attention. Ce n'est qu'au cours des dernières années qu'ont voit paraitre des travaux comparatifs, qui essayent d'étudier les rapports entre les deux dénominations, leurs conflits et leurs convergences. Le petit livre de Brian Smith, universitaire nord-américain connu pour ses travaux sur l'Église catholique chilienne, nous offre une bonne synthèse des débats à ce sujet. Évitant le réductionnisme grossier des modèles inspirés des règles de fonctionnement du marché, il confronte les différentes analyses du rôle politique des deux mouvances religieuses et avance quelques hypothèses. En ce qui concerne le pentecôtisme : tout en reconnaissant le soutien que les principaux porte-parole de ces Églises ont apporté à des régimes autoritaires il plaide pour un diagnostic plus nuancé, en insistant sur les différences internes au courant évangélique, et la présence, notamment au Brésil, de tendances progressistes, certes minoritaires, mais significatives.

11 L'apport le plus intéressant du livre porte cependant sur l'évolution actuelle de l'Église catholique, qui, contrairement à ce que l'on a souvent proclamé, ne s'est pas retirée de la scène politique après le déclin des régimes d'exception et la redémocratisation des institutions dans la plupart des pays d'Amérique latine. Elle continue à s'exprimer publiquement et à intervenir dans le débat politique, notamment autour de trois thèmes : a) la défense de la justice sociale, c'est-à-dire la critique des politiques néolibérales de dérégulation des marchés et réduction des dépenses sociales, responsables de conditions « inhumaines » de vie pour la masse des pauvres du continent ; b) la défense de la morale sexuelle traditionnelle, contre le divorce, la contraception, l'avortement ou l'éducation sexuelle ; c) la défense des privilèges légaux et institutionnels du catholicisme contre la concurrence des Églises pentecôtistes. Si l'on compare les résultats de ces trois sortes d'intervention, il est évident, constate l'auteur, que l'Église a eu beaucoup plus de succès dans sa tentative d'influencer les gouvernements et les élites politiques sur les deux derniers points que sur le premier. Par contre, au niveau de sa base sociale et populaire, c'est l'inverse: le message social de l'Église passe beaucoup mieux que son discours d'ordre moral, et ses interdictions dans le domaine sexuel, que les fidèles refusent d'obéir. 
12 Dans sa conclusion, l'auteur examine trois scénarios possibles des rapports futurs entre catholicisme et pentecôtisme: le premier serait celui d'une fuite religieuse du monde, chaque confession renforçant les tendances au repli de l'autre. Malgré la tendance des nouveaux mouvements religieux de privilégier les préoccupations privées et le salut individuel de l'âme, il n'est pas probable que ce scénario s'accomplisse. Le deuxième serait celui d'une perpétuation du conflit et de la rivalité politico-religieuse des deux confessions. L'auteur insiste sur les similitudes entre les Églises catholiques et pentecôtistes, mais il est obligé de reconnaître, à contre-cœur, que l'animosité entre les deux n'est pas près de disparaître. Enfin, le troisième scénario serait celui d'une coopération des deux confessions dans un rôle prophétique de défense de la démocratie et de la justice sociale. Ce serait le cas idéal, mais l'auteur doit constater que les convergences entre les deux se font actuellement plutôt autour des positions les plus rétrogrades en tout ce qui concerne la morale sexuelle et les droits des femmes.

Comment réagissent les femmes à cette orientation conservatrice des institutions religieuses - y compris celles qui sont les plus progressistes sur les questions socioéconomiques? Il existent peu de travaux sur ces questions, malgré le fait, reconnu par nombre d'observateurs, que les femmes constituent la majorité absolue des membres, des activistes et même des dirigeantes des communautés ecclésiales de base. Il faut donc saluer le travail pionnier de l'universitaire nord-américaine Carol Drogus sur le rôle des femmes dans l'Église populaire brésilienne des dernières années, à partir d'une recherche sur le terrain à S. Miguel Paulista, dans la banlieue est de S. Paulo.

Il est évident que toutes les femmes des CEBs n'ont pas le même type de comportement socio-religieux. L'auteur distingue entre trois «types» principaux de personnalités religieuses dont le poids dans cette population est sensiblement équivalent: les "libérationistes", qui privilégient le salut collectif, l'action socio-politique, la lutte contre le capitalisme ; les traditionalistes, qui croient plutôt à la conversion personnelle et se méfient de la politique ; les «samaritaines », qui tentent de concilier la charité et la justice, l'individuel et le social. Le discours qui permet d'unifier cet univers si hétérogène culturellement est celui qui rattache l'action sociale aux valeurs « féminines » - comme la maternité - et qui associe, dans un même élan, la charité, l'amour et la justice sociale. L'étude montre qu'il est plus facile de mobiliser ces femmes des CEBs pour des mouvements sociaux - notamment les associations de quartiers pauvres - considérés comme compatibles avec les stéréotypes de genre, que pour l'action politique, perçue comme domaine « masculin ».

15 L'engagement de ces femmes pauvres dans les CEBs n'a pas sensiblement modifié leurs rôles domestiques - qui restent marqués par la domination du «chef de famille »- mais, en leur permettant une participation active dans la sphère publique, il a tout de même battu en brèche les conceptions patriarcales sur le " rôle naturel des femmes » (limité à la sphère domestique). Indépendamment de l'attitude de la hiérarchie religieuse, assez frileuse sur ce terrain, les CEBs ont permis des expériences qui ont cristallisé, au moins chez une partie de ces femmes, une attitude critique envers les rapports de genre existants. Ainsi, une minorité des femmes de tendances «libérationiste » ont créé, à $S$. Miguel Paulista, un groupe de femmes autonome, séculaire, qui se propose de discuter de questions comme la patriarcat ou la sexualité.

16 Ces questions se trouvent aussi au cœur de la variante féministe de la théologie de la libération. Le volume publié par le Centre Tricontinental de Louvain-la-Neuve animé par François Houtart inclut un texte de la théologienne brésilienne Ivone Gebara qui explique 
les objectifs de la théologie féministe de la libération : mettre en question les hiérarchies entre hommes et femmes, et la tradition patriarcale de la religion.

Où en est la théologie de la libération, après les condamnations romaines, l'essor des groupes évangéliques, la chute du mur de Berlin ? En fait, ce ne sont pas ces questions-là qui intéressent les théologiens: leur principale préoccupation continue à être, comme avant, la condition des pauvres en Amérique latine. Le conflit des dénominations, la rivalité avec les Églises pentecôtistes, qui polarisent les attentions des sociologues - mais aussi d'une partie de la hiérarchie catholique - les laissent indifférents. C'est à partir de l'option préférentielle pour les pauvres que tentent de réfléchir Gustavo Gutierrez, Leonardo Boff, Ruben Dri, Jung Mo Sung et Franz Hinkelammert, dans les essais rassemblés dans ce recueil (qui inclut aussi des écrits sur l'islam et le bouddhisme, mais ceux-là sont hors du cadre de cette note critique). Certes, on voit apparaître des interrogations nouvelles, comme l'écologie, le respect et la vénération pour la création, dont L. Boff se fait le porte-parole prophétique. Mais la plupart de ces écrits des années 1990 ont pour objet les causes de la pauvreté, et donc la critique théologique du capitalisme néolibéral, de son « idolâtrie du marché » et de sa " théologie sacrificielle ».

Il est à noter que la plupart de ces écrits font un usage extensif des sciences sociales - non seulement Marx et la théorie de la dépendance, mais aussi Max Weber, cité par Jung Mo Sung comme un témoin inattendu des méfaits du capitalisme, ce système où, selon l'auteur de l'Éthique protestante «l'homme est dominé par la production de l'argent, par l'acquisition, envisagée comme finalité ultime de sa vie». Un des théologiens, l'évangélique péruvien Pablo Barrera Riviera, va jusqu'à expliquer les difficultés de la théologie de la libération par la crise des paradigmes dans les sciences sociales, ce qui est quand même un peu excessif.

19 En tout cas, ce recueil témoigne de la vitalité de la théologie de la libération comme phénomène culturel et socio-religieux, dont on avait, au début des années quatre-vingtdix, annoncé un peu trop vite la disparition.

\section{AUTEUR}

\section{MICHAEL LÖWY}

Centre d'Études Interdisciplinaires des Faits Religieux - C.N.R.S. 